\title{
ÉDITORIAL
}

\section{Les plantes adaptées à des désordres inadaptés}

\author{
P. Goetz \\ (C) Lavoisier SAS 2016
}

Il y a quelques années, j'assistais à une formation continue présidée par le Pr Kuntzmann, rhumatologue. En réalité, celui-ci éveillait l'attention des auditeurs par les voies nouvelles qui étaient issues de la recherche fondamentale en rhumatologie. Nous apprîmes que ce que nous appelions « arthrite » était un phénomène très complexe dont la physiopathologie s'éloignait très sensiblement de la rhumatologie primaire que nous connaissions. On y voyait en premier comment la génétique entrait par des excès ou des insuffisances dans le déclenchement d'une inflammation articulaire. Et l'on parla du système HLA-B27. Par ailleurs, le schéma classique des prostaglandines et de l'acide arachidonique était un modèle suranné. C'était une image vue à la loupe alors qu'il fallait passer au microscope électronique. Qui dit rhumatologie et système tissulaire ne peut que rajouter : immunologie. La défense de l'organisme avec ses défauts qui conduisent à l'élaboration d'autoanticorps. Audelà, le système tissulaire et le système circulatoire fournissent des médiateurs : les kinases, les TNF- $\alpha$, mais aussi le natural killer, le NF-кB (nuclear factor-kappa B); ensuite, les médiateurs classiques que sont les interleukines, et finalement les COX et LOX, et les PGE, sans oublier les éléments qui assurent le signalement et favorisent la mise en route du système.

À ce niveau-là, on comprend facilement le pont qui relie une infection « banale » à virus qui a une inflammation articulaire ou en altération du système neurologique. Ce que l'on appelait maladies systémiques entre dans le grand cadre de ces pathologies tissulaires immunologiques. Aujourd'hui, on rencontre facilement un patient connu et traité pour une arthrite inflammatoire qui, dix ans après, se trouve aussi avoir une rectocolite ulcérohémorragique. Enfin, plus personne ne s'étonne d'une arthrite psoriasique, alors qu'on lie surtout le psoriasis à un trouble psychiatrique même peu prononcé. La relation et le terrain immunologique défaillant ne sont plus étonnants chez un patient avec une affection psychiatrique.

Or, depuis 35 ans de phytothérapie, ces phénomènes étaient connus à travers les expérimentations et constatations cliniques. Souvent, on s'apercevait qu'une drogue végétale pouvait renforcer l'organisme, agir sur la défense infectieuse immunologique et en même temps améliorer des pathologies rhumatismales ou dermatologiques. La majorité des drogues adaptogènes agissent dans ce sens.

Nous avons choisi comme matière médicale la schisandre, la "graine aux cinq saveurs ", une plante adaptogène qui a une action pharmacologique sur TNF- $\alpha$, le natural killer, les Il-1 et Il-6, COX et LOX, les PGEs et le taux de NO.

Au total, il faut oublier la notion de médecine holistique, mais se rendre compte des phénomènes physiopathologiques de la neuro(psycho)-immunorhumatologie (Tableau 1). Notre conception verticale des indications d'une plante doit laisser la place à une conception horizontale d'effets avec, en outre, les synergies des plantes entre elles, mais aussi avec la biothérapie.

C'est maintenant à la génération des futurs professionnels de la phytothérapie de développer ce grand concept, que j'explique dans ce numéro, sous le titre « Nouveaux aspects de la phytothérapie en rhumatologie : plantes et neuroimmunorhumatologie $»^{1}$.
P. Goetz $(\bowtie)$

Dumenat de phytothérapie, Paris-XIII,

F-93017 Bobigny cedex, France

e-mail : paul.goetz@wanadoo.fr

\footnotetext{
${ }^{1}$ Conférence donnée lors des « Rencontres de phytothérapie de Courbevoie ».
} 
Tableau 1 Pathologies inflammatoires, HLA-B27 et pathologies auto-immunes.

\begin{tabular}{|c|c|c|c|c|c|c|c|c|}
\hline $\begin{array}{l}\text { Plantes } \\
\text { médicinales }\end{array}$ & Rhumatologie & Uvéite & $\begin{array}{l}\text { S. } \\
\text { o.u.synv. }\end{array}$ & $\begin{array}{l}\text { RCHU } \\
\text { Colite ulc. }\end{array}$ & Dermatose & Inflammation & Psychiatrie & Cancérologie \\
\hline Curcuma longa & $\mathrm{xx}$ & $\mathrm{x}$ & & $\mathrm{xx}$ & $\mathrm{xx}$ & $\mathrm{xxx}$ & & \\
\hline Boswellia serrata & $\mathrm{xxx}$ & & & $\mathrm{xx}$ & $\mathrm{xx}$ & $\mathrm{xxx}$ & & \\
\hline Sarsaparilla & $\mathrm{x}$ & & & & $\mathrm{xx}$ & $\mathrm{x}$ & & \\
\hline Urtica d./ u. & $\mathrm{xx}$ & & & $\mathrm{xx}$ & & $\mathrm{x}$ & & \\
\hline Harpagophytum & $\mathrm{xx}$ & & & $\mathrm{xx}$ & & $\mathrm{xxx}$ & & \\
\hline Trypterigium & $\mathrm{xx}$ & & $\mathrm{xx}$ & & & & & \\
\hline $\begin{array}{l}\text { Commiphora } \\
\text { myrrha }\end{array}$ & $\mathrm{xx}$ & & & $\mathrm{xx}$ & & $\mathrm{xx}$ & & \\
\hline Echinacea & $\mathrm{x}$ & & & $\mathrm{xx}$ & $\mathrm{xx}$ & $\mathrm{xx}$ & & Infectiologie \\
\hline $\begin{array}{l}\text { Uncaria } \\
\text { tomentosa }\end{array}$ & $\mathrm{xx}$ & & & & & $\mathrm{x}$ & & $\mathrm{x}$ \\
\hline Oméga $3 \& 6$ & $\mathrm{xx}$ & & & $\mathrm{x}$ & & $\mathrm{xx}$ & $\mathrm{xx}$ & \\
\hline
\end{tabular}

